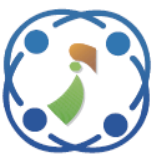

\title{
Medical Image Fusion Using Integrated Guided Nonlinear Anisotropic Filtering with Image Statistics
}

\author{
Mummadi Gowthami Reddy ${ }^{1 *}$ \\ Palagiri Veera Narayana Reddy ${ }^{2}$ \\ Patil Ramana Reddy ${ }^{1}$ \\ ${ }^{I}$ Department of Electronics and Communication Engineering, \\ Jawaharlal Nehru Technological University College of Engineering, Anantapur, Andhra Pradesh, India \\ ${ }^{2}$ Department of Electronics and Communication Engineering, \\ Sri Venkateswara College of Engineering, Kadapa, Andhra Pradesh, India \\ * Corresponding author's Email: mgowthamireddy25@ gmail.com
}

\begin{abstract}
An image that is highly informative is produced by unifying the information from couple of or numerous source images which is referred as image fusion. It has been employing in most of the applications in the medical field like detecting of tumours, treating Alzheimer's, surgery of brain with the assistance of computer and some other clinical diagnosis. Successful diagnosis of specific diseases necessitates the enhancement in the exactness of fusion algorithms. For examining the body of human, the images obtained from magnetic resonance imaging (MRI) and computed tomography (CT) plays a vital role. Channelizing the maximum info from the source images to the fused image with understate loss of info that must mitigate the presence of artifacts in the fused outcome is the basic idea of any fusion methodology. In this context, a novel medical image fusion approach is implemented, that utilizes integrated guided and nonlinear anisotropic (IGNLA) filtering with image statistics. This approach upholds the info of texture in the fused images more efficaciously. In addition, proposed medical image fusion is extended for color images and applied to MR-Gad, MR-T2 and SPECT-Tc images. Extensive simulation results of proposed medical image fusion are compared with traditional and recent image fusion algorithms and disclose the superiority of proposed approach with respect to image quality metrics.
\end{abstract}

Keywords: MR and CT imaging, SPECT imaging, Image fusion, Edge preservation, Texture preservation, Guided image filtering, Non-linear anisotropic filtering, Image statistics and Image quality metrics.

\section{Introduction}

Various combinations of sensed images are handled by image fusion, where these sensed images are acquired from various sensors that admit high and multi spectrum, viewing at multiple angles and resolutions, which improves assess for achieving the image quality. There are wide range of fields which utilizes multi-sensor images and that includes medical imaging, remote sensing and computer vision. Fusion of medical images renders good informative image for clinical assessment since the multi-model fused image has more significant information than a single image and it is a combining procedure of compliment fusion methodologies for clinical assessment. In practice, there are different types of multi-modality medical images like magnetic resonance imaging (MRI), computed tomography (CT) and single photon emission computerized tomography (SPECT or SPET) [1, 2] etc. which assists the physician to handle with more precise clinical diagnosis and assessment. For instance, dense structures such as bones and implants with lesser deformation can be obtained by CT imaging but the changes in physiological information cannot be detected in CT, where the MRI is capable to obtain the information about normal and pathological soft tissues without having the structures of bone and implants. Similarly, SPECT is a technique of nuclear medicine tomographic imaging employed by gamma rays and 


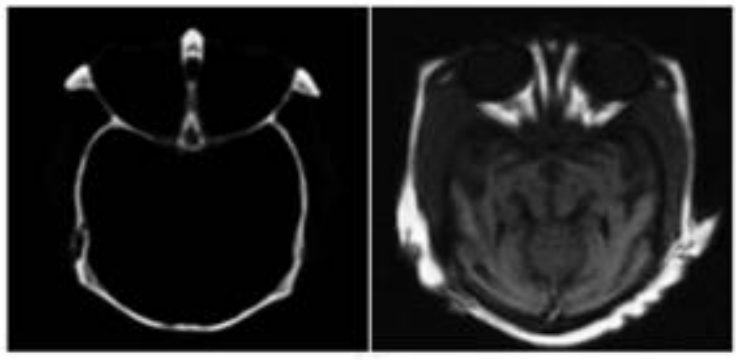

(a)

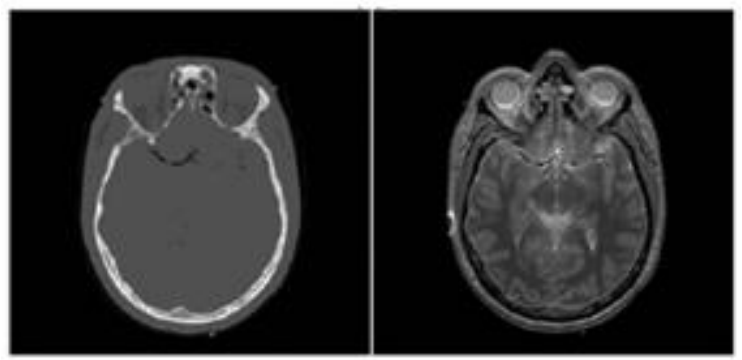

(b)

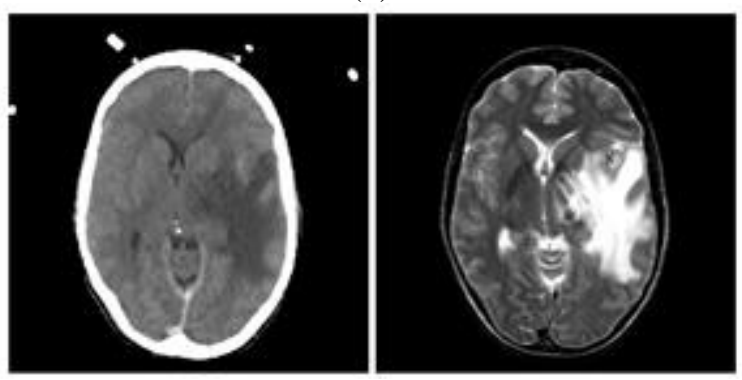

(c)

Figure.1 CT and MR images: (a) dataset 1, (b) dataset 2, and (c) dataset 3

acquires multiple two-dimensional images (alsoknown as projections) from multiple angles. Afterwards, a tomographic reconstruction is employed using the computer to get a threedimensional dataset. However, this dataset may then be altered to disclose thin slices along any chosen axes of body. By considering the imaging techniques like MRI, CT and SPECT or SPET, a physician can't obtain a precise clinical need for further diagnosis with a single image. Thus, it is essential to fuse the medical images with multimodalities and this has become very promising and highly challenging task in the field of medical research in recent years [3].

Representing the ocular information by two or more images into a solitary image without losing original data is referred as fusion of images [4], where the ambiguity associated to a single image can be mitigated by the process of fusion. In recent days, obtaining human's anatomies and functions with high resolution and more instructive description becomes potential due to advancement in the field of medical imaging technology. The encouragement for the research in the analysis of medical images has been done by such development. In addition, the development of medical images vitality in the clinical applications rendered a straight effect on this field of research [5]. From the past decades, there has been a greater number of scientific research papers have been published on the topic of fusing the medical images. Essentially, image fusion techniques have been classified into three sorts. They are pixel level, feature level and decision level. Successful fusion methods based on morphological operators are discussed in [6-7].

However, the fused images of these approaches suffering from lack of visualization in spite of their simplicity. Later authors in [8, 9] addressed optimization-based fusion algorithms, where the issue of optimization is referred as Bayesian, which is quite hard to resolve in practice. This problem can be resolved by calculating edge coordinated weights and those methods were cited as Markov random field (MRF) [10] and generalized random walk [11]. But due to the numerous iterations, fused image might be over smoothed. Later, researches focused on artificial neural networks (ANNs) $[12,13]$, which pulls lots of interest in the era of fusion. Generally, these approaches are motivated from the fusion of biological signals. However, since the human visual system (HVS) is sensitive to the info of edges, multiresolution transform-based approaches are implemented. Pyramid decomposition and wavelet transform are adjunct to this class. Gradient and Laplacian pyramid $[14,15]$, low-pass pyramid ratio [16], Gaussian pyramid [17] and morphological pyramid [18] are the several fusion approaches under the class of pyramid decomposition where source image gets decomposed into lowpass filtered subbands. Every subband image represents the source image info at various scales.

Wavelet transform based fusion algorithms like discrete wavelet transform (DWT) [19, 20], stationary wavelet transform (SWT) [21] have tremendous performance over the algorithms presented in the literature. Recent years, many extended versions of wavelet transform have done to improve the fusion performance further [22-25]. However, artifacts might be innovated with this sort of fusion algorithms. In addition, visual quality of fused image is not good enough for further diagnosis. Next, optimization techniques have been introduced to surmount these issues, which considers numerous iterations to obtain fused image that results in over smoothing. Principal component analysis (PCA) is one of the finest methodologies for obtaining fused images from given MR and CT images [26]. But, PCA based approaches over smoothen the fused image due to multiple iterations there by texture and 
edge information might lost. In summation, now a day fusion approaches those preserves the edge information are becoming more popular which employ smoothing process for fusing of images. Guided image filter [28], weighted least square filter [29-30], bilateral filter [31], cross bilateral filter [32], 3-D anisotropic diffusion [33] based approaches are the more popular fusion algorithms in this class. Majority of above said algorithms decompose the source image into the layers of approximate or base and detail. One can obtain the fused image by altering or modifying the approximate or detail or even both layers. But the gradient reversal artifacts and halo effects were rendered during the fusion process using bilateral or cross bilateral and guided filter respectively. Practically, there are three major attributes to be concerned for an effective fusion of medical images.

1) Maximum exchange of meaningful information from input images to the fused image.

2) While doing fusion, there shouldn't any loss of information.

3) Artifacts and any additional info shouldn't be innovated into the fused image.

Therefore, to resolve the issues of conventional fusion methodologies, a novel integrated guided non-linear anisotropic filtering with image statistics is introduced in this article. Further, the proposed approach is implemented by considering the attributes mentioned above. In this, filtering of every source image is done by employing IGNLA filtering, that extracts the layers of approximate and detail. Then image statistics based fusion rule is applied to get fused image. The novel contributions of this work can be summarized as follows:

- A Novel utilization of guided non-linear anisotropic filtering for extracting the features from the input MR, CT and SPECT images to be fused. As per the authors best knowledge, this combo hasn't utilized in image fusion applications yet.

- A Fully new fusion framework is introduced based on proposed hybrid methodology. We mainly focused on the retaining of texture even after fusion process. Algorithms presented in the literature like discrete wavelet transform (DWT) [20], stationary wavelet transform (SWT) [21], principal component analysis (PCA) [26], NLAF-PCA [34], SWT+PCA [35] and fast discrete curvelet transform (FDCT) [27] aimed at enhancement in the performance of fusion process only. These methodologies haven't concentrated on the visual texture of fused medical images.
- We considered several quality indexes to disclose the effectiveness and robustness of proposed IGNLA filtering with image statistics fusion model.

- Further, it is applied to MR-Gad, MR-T2 and SPECT-Tc images to demonstrate the effectiveness of proposed fusion methodology towards RGB image fusion.

Rest of the paper structure is as follows: section II describes the NLAF briefly. Section III presents the proposed fusion framework. Section IV presents the results and discussion. Section V includes the conclusions and future enhancements.

\section{Nonlinear anisotropic filtering}

This section explains the procedure of NLAF that smooths the source medical image into the regions of homogeneous as maintaining the edge information (non-homogeneous regions) by employing partial differential equations (PDE). This NLAF is an extended version of non-linear isotropic filtering, where the information at nonhomogeneous regions is lost due to the smoothing at inter-regions. In contrast, NLAF produces the images of coarser resolution by utilizing intra-region smoothing, where every edge at coarser resolution is sharp and useful. Usually, a flux function is utilized for controlling the diffusion of an image $I$ as

$$
I_{t}=\mathbb{F}(x, y, t) \Delta I+\nabla \mathbb{F} \cdot \nabla I
$$

Where $\mathbb{F}(x, y, t)$ is flux function, $\Delta$ is a Laplacian operator, $\nabla$ is a gradient operator and $t$ is time or scaling constant.

Eq. (1) can also be named as a heat equation and can be resolved by employing forward time central space approach. The solution for this PDE is

$$
\begin{aligned}
& \quad I_{i, j}^{t+1}=I_{i, j}^{t}+\beta\left[\mathbb{F}_{N} \cdot \bar{\nabla}_{N} I_{i, j}^{t}+\mathbb{F}_{S} \cdot \bar{\nabla}_{S} I_{i, j}^{t}+\mathbb{F}_{E} .\right. \\
& \left.\bar{\nabla}_{E} I_{i, j}^{t}+\mathbb{F}_{W} \cdot \bar{\nabla}_{W} I_{i, j}^{t}\right]
\end{aligned}
$$

In above eq., $I_{i, j}^{t+1}$ is the coarser resolution image at $t+1$ scale which depends on the previous coarser scale image $I_{i, j}^{t} . \quad \beta$ is a stability constant satisfying $0 \leq \beta \leq 1 / 4$. Nearest neighbour differences in north, south, east and west directions denoted as $\bar{\nabla}_{N}$, $\bar{\nabla}_{S}, \bar{\nabla}_{E}, \bar{\nabla}_{W}$ respectively. They are defined as

$$
\begin{aligned}
& \bar{\nabla}_{N} I_{i, j}=I_{i-1, j}-I_{i, j} \\
& \bar{\nabla}_{S} I_{i, j}=I_{i+1, j}-I_{i, j} \\
& \bar{\nabla}_{E} I_{i, j}=I_{i, j+1}-I_{i, j}
\end{aligned}
$$




$$
\bar{\nabla}_{W} I_{i, j}=I_{i, j-1}-I_{i, j}
$$

Similarly, the flux functions are denoted as $\mathbb{F}_{N}$, $\mathbb{F}_{S}, \mathbb{F}_{E}$ and $\mathbb{F}_{W}$ respectively.

$$
\begin{aligned}
& \mathbb{F}_{N_{i, j}}^{t}=g\left(\left\|(\nabla I)^{t}{ }_{i-1 / 2, j}\right\|\right)=g\left(\left|\bar{\nabla}_{N} I_{i, j}^{t}\right|\right) \\
& \mathbb{F}_{S_{i, j}}^{t}=g\left(\left\|(\nabla I)^{t}{ }_{i+1 / 2, j}\right\|\right)=g\left(\left|\bar{\nabla}_{S} I_{i, j}^{t}\right|\right) \\
& \mathbb{F}_{E_{i, j}}^{t}=g\left(\left\|(\nabla I)^{t}{ }_{i, j+1 / 2}\right\|\right)=g\left(\left|\bar{\nabla}_{E} I_{i, j}^{t}\right|\right) \\
& \mathbb{F}^{t}{ }_{W_{i, j}}=g\left(\left\|(\nabla I)^{t}{ }_{i, j-1 / 2}\right\|\right)=g\left(\left|\bar{\nabla}_{W} I_{i, j}^{t}\right|\right)
\end{aligned}
$$

In Eqs. (7)-(10), $g(\cdot)$ is a consistently decreasing and never increasing function with $g(0)=1$ and dissimilar operations can be utilized for $g(\cdot)$. But couple of functions are advised by authors in [36] as described below:

$$
\begin{aligned}
& g(\nabla I)=e^{-\left(\frac{\|\nabla I\|}{k}\right)^{2}} \\
& g(\nabla I)=\frac{1}{1+\left(\frac{\|\nabla I\|}{k}\right)^{2}}
\end{aligned}
$$

The above functions provide a trade-off between the preservation of smoothing and texture where the primary one is serviceable if there are edges with higher contrast over lower contrast while the next is useful if there are wider regions over the smaller. Both functions comprise of a parameter denoted with $k$, which is utilized to conclude the region boundary validity depending on its strength of edge.

\section{Proposed methodology}

This section describes the brief explanation of our proposed IGNLA filtering fusion framework. Fused output image is obtained by implementation of guided filtering process to obtain the approximate and detail layers with NLAF followed by a fusion rule based on image statistics. Our proposed fusion methodology is depicted in Fig. 2.

\subsection{Guided filter}

The main basis of guided filter is first, blur the input images to be fused, then these images are subtracted from the representing input images to obtain the images those are sharp. Finally, the

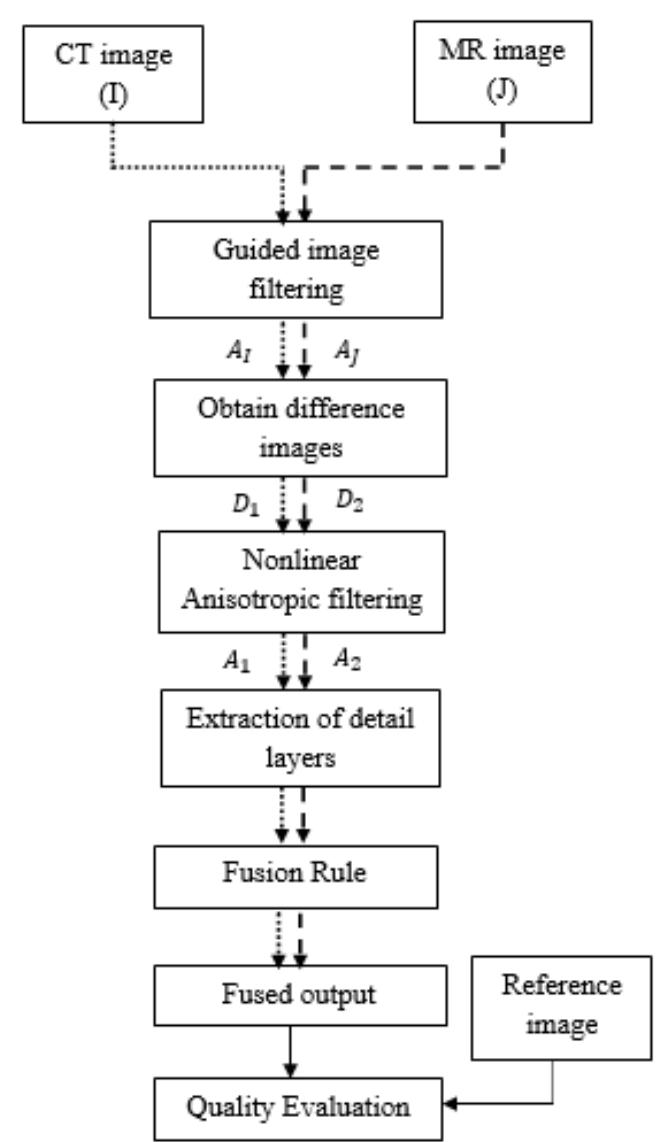

Figure. 2 Proposed fusion process flow using IGNLA filtering with image statistics

weighted coefficients are computed by utilizing these sharpened image details for fusion process. Authors simply explained this approach with the images accompaniment by separate descripting the interpolates in each stage for better analysis. The proposed guided filter method is explained as follows:

Assume that the guided image is denoted as $g$ which is riveted at a picture element $m$ inside a window $w$ that is local square, then the output $(\mathcal{O})$ of guided filter at a pixel $n$ is expressed as:

$$
\mathcal{O}_{n}=a_{m} g_{n}+b_{m}, \forall n \in w_{m}
$$

Where $a_{m}$ and $b_{m}$ denoted as constant linear coefficients in window $w_{m}$. To find out linear coefficients, constraints must be inferred from the source image $\mathcal{J}$. Now, the subtraction of undesired components $(\mathcal{U})$ from the source image is done to get the output which is noiseless.

$$
\mathcal{O}_{n}=\mathcal{J}_{n}-\mathcal{U}_{n}
$$

The solvent for this issue should diminish the divergence between the input $(\mathcal{J})$ and filtered output $(\mathcal{O})$ and should also preserve the relation in Eq. (14). 
Therefore, $a_{m}$ and $b_{m}$ are the coefficients those are linear and can the operation of cost in window $w_{m}$ is diminished as

$$
\begin{aligned}
& E\left(a_{m}, b_{m}\right)=\sum_{n \in w_{m}}\left(\left(a_{m} g_{n}+b_{m}-\mathcal{J}_{n}\right)^{2}+\right. \\
& \left.\quad \mathfrak{r} a_{m}{ }^{2}\right)
\end{aligned}
$$

Where the parameter of regulization denoted as $\mathfrak{r}$ and the above equation constitutes the model of linear regression. Expression for resolving this is given as

$$
\begin{aligned}
& a_{m}=\frac{\frac{1}{|w|} \sum_{n \in w_{m}} g_{n} \jmath_{n}-\mu_{m} \bar{\jmath}_{n}}{\sigma_{m}^{2}+\mathfrak{r}} \\
& b_{m}=\overline{\mathcal{J}}_{n}-a_{m} \mu_{m}
\end{aligned}
$$

Here, pixel quantity in a window $w_{m}$ riveted at picture element $m$ denoted as $|w|, \mu_{m}$ is the mean, and $\sigma_{m}^{2}$ is the variance in the window $w_{m} \cdot \overline{\mathcal{J}}_{n}$ is the mean of input $J_{n}$ in $w_{m}$ and is given by

$$
\overline{\mathcal{J}}_{n}=\frac{1}{w} \sum_{n \in w_{m}} \mathcal{J}_{n}
$$

The filtered output $\left(\mathcal{O}_{n}\right)$ can be resolved once the achievement of linear coefficients is done according to the Eq. (14). But various imbrication windows $\left(w_{m}\right)$ which are centered at $m$ comprise of pixel $n$ in common. Now, compute the aggregate of all estimation of filtered output $\left(\mathcal{O}_{n}\right)$ to resolve this issue. Therefore, $\mathcal{O}_{n}$ can be expressed as

$$
\mathcal{O}_{n}=\overline{a_{m}} g_{n}+\overline{b_{m}}
$$

Where linear coefficients aggregates are denoted as $\overline{a_{m}}=\frac{1}{w} \sum_{n \in w_{m}} a_{m}$ and $\overline{b_{m}}=\frac{1}{w} \sum_{n \in w_{m}} b_{m}$.

Here, output filtered image of guided image (J) in the guiding of $g$ is denoted as $\mathcal{G}_{n, \mathrm{r}}(\mathcal{J}, \mathcal{g})$, where size of filter and smoothing degree or parameter of regulization is denoted by $n$ and $\mathfrak{r}$ respectively, which controls the guided filter behaviour. The pixel in the window centre remains unaltered when there is a higher variance $\left(\sigma^{2} m\right)$ of guided image over the threshold $\mathfrak{r}$ value i.e., $\left(\sigma_{m}^{2} \geq \mathfrak{r}\right)$, whereas the pixel is substituted by the locality mean.

\subsection{Extraction of approximated and detail layers from guided filtered images using NLAF}

Let the difference images of guided filtered output $\mathcal{G}_{n, \mathrm{r}}(\mathcal{J}, \mathcal{g})$ of MR and CT images with their source images are denoted as $\mathcal{G}_{1}(x, y), \mathcal{G}_{2}(x, y)$

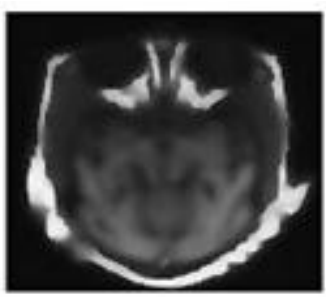

(a)

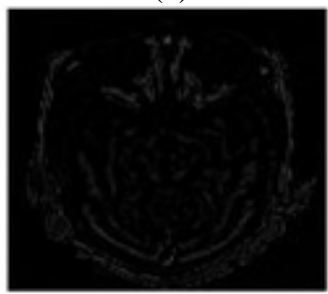

(c)

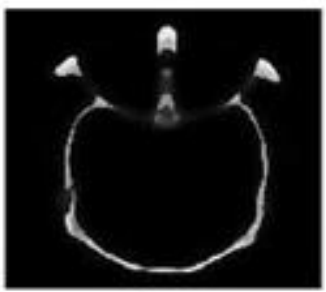

(b)

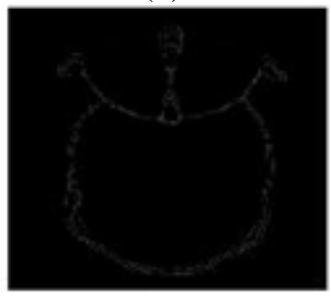

(d)
Figure.3 (a) approximate layer of MR image, (b) approximate layer of CT image, (c) detail layer of MR image, and (d) detail layer of CT image

respectively with a size of $p \times q$ and these two images are co-registered images. As shown in Fig. 2, these two guided filtered images are passed through the NLAF block to obtain the approximate layers.

$$
\begin{aligned}
& A_{\mathcal{G}_{1}}(x, y)=\operatorname{nlaf}\left(\mathcal{G}_{1}(x, y)\right) \\
& A_{\mathcal{G}_{2}}(x, y)=\operatorname{nlaf}\left(\mathcal{G}_{2}(x, y)\right)
\end{aligned}
$$

Where $A_{\mathcal{G}_{1}}(x, y)$ and $A_{\mathcal{G}_{2}}(x, y)$ are $n^{t h}$ approximate layers and nlaf is a sub function that process the source image (refer section II for more information). Now, the detail layers are obtained by subtracting the output of NLAF by utilizing Eqs. (13) and (14).

$$
\begin{aligned}
& D_{\mathcal{G}_{1}}(x, y)=\mathcal{G}_{1}(x, y)-A_{\mathcal{G}_{1}}(x, y) \\
& D_{\mathcal{G}_{2}}(x, y)=\mathcal{G}_{2}(x, y)-A_{\mathcal{G}_{2}}(x, y)
\end{aligned}
$$

Fig. 3 demonstrates that the output layers obtained from IGNLA filtering process i.e., approximation and detail layers of MR and CT images.

\footnotetext{
Algorithm: IGNLA filtering with image statistics based fusion process

Step 1: Select and read MR and CT source images from the MATLAB current directory (data set2 shown in Fig. 1).

Step 2: Convert the source images into gray scale in case of RGB images.

Step 3: Apply guided filter to obtain the guided images of MR and CT images as described in section 3.1.

Step 4: Now, obtain the difference images by subtracting the guided filtered output images form their source MR and CT images.
}

Step 5: Apply NLAF process to the difference images 
obtained from Step 4 to get the approximate and detail layers as shown in Fig. 3.

Step 6: Compute the covariance of detail layers obtained from step 5.

Step 8: Calculate the Eigen vectors for step 6 output.

Step 9: Now, apply fusion rule to obtain final fused output of MR and CT images.

\subsection{Fusion rule}

Here fusion rule functions with the computation of weight representing to a pixel based on the edge strengths in the direction of horizontal and vertical. In theory, to determine a weight representing to a picture element at a position $(m, n)$ in a medical image assume a window $w$ of square with a length of $p \times p$ about its locality. Take $\mathcal{Q}$ as a matrix and compute the matrix of its covariance by taking row and column as a reflexion and variable severally.

$$
\operatorname{cov}(\mathcal{Q})=E\left[(\mathcal{Q}-E[Q])(Q-E[Q])^{T}\right]
$$

Calculate unbiased horizontal estimate of a covariance matrix at $(m, n)$ location of pixel as

$$
\mathfrak{U}_{\mathcal{E}_{\mathrm{H}}}^{m, n}(Q)=\frac{1}{p-1} \sum_{k=1}^{p}\left(Q_{k}-\overline{\mathcal{Q}}\right)\left(Q_{k}-\overline{\mathcal{Q}}\right)^{T}
$$

Where $\mathcal{Q}_{k}$ is denoted as $p$-dimensional variable's $k^{\text {th }}$ observation and $\overline{\mathcal{Q}}$ is the observation's mean. Interestingly diagonal of $\mathfrak{U}_{\mathcal{E}_{\mathrm{H}}}^{m, n}(\mathcal{Q})$ is a row matrix of variance. Formulate the esteem of Eigen $\lambda_{\mathcal{E}_{\mathrm{H}}}^{h}$ of $\mathfrak{U}_{\mathcal{E}_{\mathrm{H}}}^{m, n}(\mathcal{Q})$. Since the matrix length is $p \times p$ the retrieved quantity of Eigen esteems also $p$. Now, accumulate all the potential esteems of Eigen to acquire the edge strength $\mathbf{E}_{\varepsilon_{\mathrm{H}}}$ in the direction of horizontal.

$$
\mathrm{e}_{\varepsilon_{\mathrm{H}}}(m, n)=\sum_{h=1}^{p} \lambda_{\varepsilon_{\mathrm{H}}^{k}}^{k}
$$

Likewise, reverse the process for edge strengths in vertical direction of by considering column and row as observation and variable respectively. Compute the unbiased vertical estimate $\mathfrak{H}_{\mathcal{E}_{v}}^{m, n}$, and then calculate the Eigen values $\lambda_{\mathcal{E}_{v}}^{h}$. Now, the edge strength in the direction of vertical $e_{\mathcal{E}_{\mathcal{V}}}$ can be acquired by totalling these esteems of Eigen as,

$$
\mathrm{e}_{\varepsilon_{\mathcal{V}}}(m, n)=\sum_{k=1}^{p} \lambda_{\mathcal{E}_{\mathcal{V}}}^{k}
$$

Now, compute the total of $\mathbf{e}_{\mathcal{E}_{\mathrm{H}}}(m, n)$ and $\mathrm{e}_{\mathcal{E}_{v}}(m, n)$ to determine the picture element weight $\mathbb{W}(m, n)$ at a position $(m, n)$.

$$
\mathbb{W}(m, n)=\mathbf{e}_{\mathcal{E}_{\mathrm{H}}}(m, n)+\mathbf{e}_{\mathcal{E}_{\mathcal{V}}}(m, n)
$$

Adaptive weights will be assigned by iterating this process for every pixel presented in an image, where the pixel weight relay on the edge strength not on the value of intensity.

\section{Results and discussion}

All the experiments have been done in MATLAB 2018a version under the high-speed CPU conditions for faster running time. The images disclosed in Fig. 1, which are cited as dataset 1, dataset 2 and dataset 3 are utilized for testing the proposed and existing fusion approaches. In general, integration of necessitated data from couple of source images into the fused image is the objective of any fusion approach and the quality of fused image cannot be judged entirely by viewing it or by assessing the quality metrics. It should be evaluated both qualitatively and quantitatively by perceptual analysis and fusion metrics. This section presents both visual quality and quantitative analysis of various algorithms such as, Wavelet based methods discrete wavelet transform (DWT) [20], stationary wavelet transform (SWT) [21], NLAF-PCA [34], stationary wavelet transform with principal component analysis (SWT+PCA) [35] and fast discrete Curvelet transform (FDCT) [27]. Analysis of fusion metrics along with image quality assessment (IQA) metrics such as peak signal-tonoise ratio (PSNR), structural similarity index (SSIM), correlation coefficient (CC), root mean square error (RMSE), entropy and mutual information (MI) are considered to verify the effectiveness of the proposed algorithm. For better quality, fused image should have optimal values for all these metrics. The fusion metric with best value is highlighted in bold letter. Visual quality of fused images obtained using state-of-art algorithms and our method has demonstrated in Figs. 4 and 5 with data set 1 and data set 2 . We can clearly observe that the perceptual quality of fused output using PCA, shown in Fig. 4 (a) looks low resolute image and the gray levels hasn't up to the mark. Other transformation methods like DWT [20], SWT [21] and the algorithm demonstrated in [27] shown in Fig. 4 (b)-(d) respectively, which performed superior to the PCA method in terms of visual perception, however these methods suffer from lack of contrast and edge preservation. Fig. 4 (e) shown that the fused output of the method presented in [35], which was far better than the above-mentioned algorithms. NLAF-PCA method which is presented in Fig. 4 (f), 


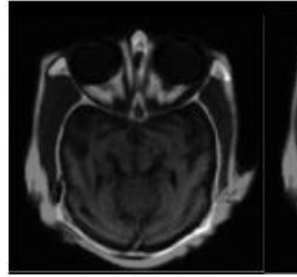

(a)

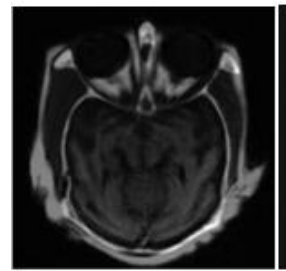

(d)

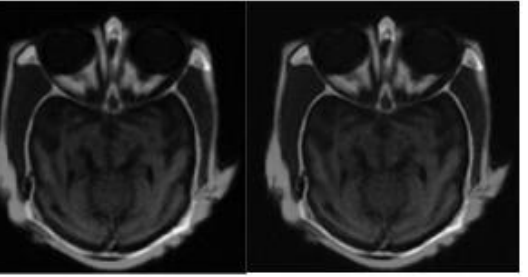

(b)

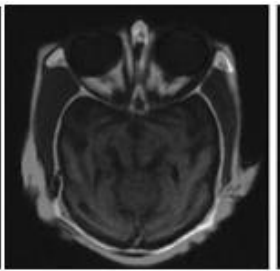

(e)

(g) (c)

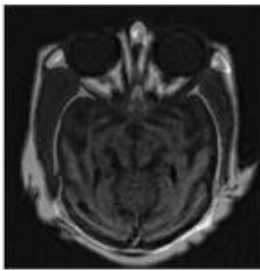

(f)

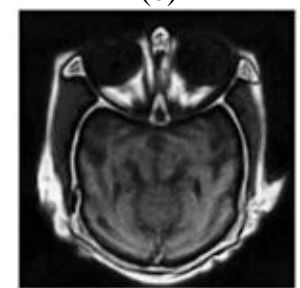

Figure.4 Visualization of fused output images with dataset 1: (a) PCA, (b) DWT, (c) SWT, (d) FDCT, (e)

SWT+PCA, (f) NLAF-PCA, and (g) Our method

which looks far better in visualization, good contrast with somewhat edge information and moderate texture preservation as the value of entropy is much higher over existing fusion methods. However, all the existing fusion methods outputs not good at visual perception, lack of contrast with edge information and texture preservation.

Figs. 5 (a)-(g) demonstrated that the PCA, DWT, SWT, FDCT, SWT+PCA, NLAF-PCA and our proposed method fused outputs with data set 2 . The same analysis which we have discussed above has applicable for this also. Quantitative analysis with IQA sown in Table 1 for the test results presented in Fig. 4, which gives the analysis of dataset 1 . Table 1 consists of various fusion metric parameters such as PSNR, RMSE, CC, SSIM, entropy and MI. The best values are highlighted in bold letters. Our proposed

method obtained far better values over all the existing fusion methods discussed in the literature. We also tested the qualitative analysis of dataset 2 and dataset 3 with the similar fusion metric parameters considered for dataset 1 and demonstrated in Tables 2 and 3 respectively. Further, Figs. 6 and 7 disclose the obtained fused results of proposed fusion approach with RGB images. Fig. 6 shows the MR-T2 and SPECT-Tc image with their corresponding fused image. Fig. 7 demonstrates the Mr-Gad and SPECT-Tc images with their fused image respectively. Table 4 shows the quality assessment of RGB fused images, where entropy, mean, standard deviation and execution time are assumed as a quality metrics.

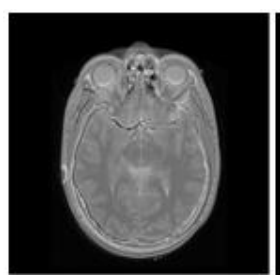

(a)

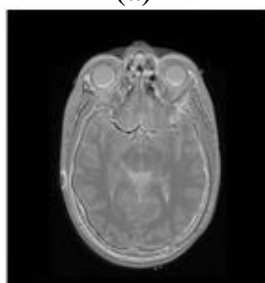

(d)

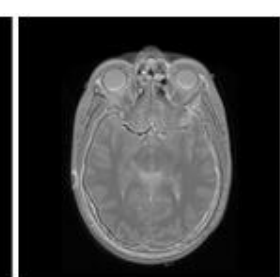

(b)

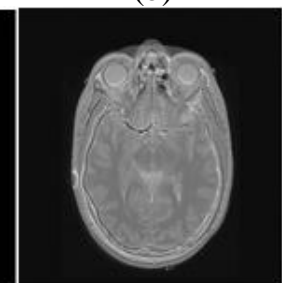

(e)

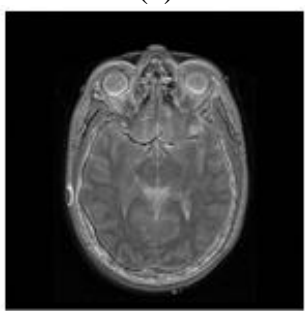

(g)

Figure. 5 Visualization of fused output images with dataset 2 (a) PCA (b) DWT (c) SWT (d) FDCT (e) SWT+PCA (f) NLAF-PCA (g) Our method

Table 1. Quantitative analysis of fusion methods for dataset 1

\begin{tabular}{ccccccc}
\hline Methodology & PSNR (in dB) & RMSE & CC & SSIM & Entropy & MI \\
\hline PCA [26] & 59.4 & 0.27 & 0.936 & 0.9908 & 6.1 & - \\
SWT [21] & 62.253 & 0.1967 & 0.7928 & 0.986 & 6.11 & - \\
DWT [20] & 62.257 & 0.1966 & 0.7935 & 0.986 & 6.099 & - \\
FDCT [27] & 65.156 & 0.146 & 0.9 & 0.9983 & 5.963 & - \\
SWT+PCA [35] & 64.96 & 0.143 & 0.9 & 0.997 & 6.19 & - \\
NLAF-PCA [34] & 65.06 & 0.142 & 0.913 & 0.997 & 6.24 & - \\
Our method & $\mathbf{8 6 . 7 4}$ & $\mathbf{0 . 0 1 1}$ & $\mathbf{0 . 9 9 8 7}$ & $\mathbf{0 . 9 9 9 9}$ & $\mathbf{7 . 0 2}$ & $\mathbf{6 . 9 9}$ \\
\hline
\end{tabular}


Table 2. Quantitative analysis of fusion methods for dataset 2

\begin{tabular}{ccccccc}
\hline Methodology & PSNR (in dB) & RMSE & CC & SSIM & Entropy & MI \\
\hline PCA [26] & 56.31 & 0.38 & 0.9786 & 0.979 & 4.31 & - \\
SWT [21] & 68.95 & 0.0909 & 0.933 & 0.988 & 4.33 & - \\
DWT [20] & 68.98 & 0.0906 & 0.934 & 0.988 & 4.34 & - \\
FDCT [27] & 70.215 & 0.140 & 0.9206 & 0.9983 & 5.05 & - \\
SWT+PCA [35] & 72.932 & 0.047 & 0.96 & 0.999 & 4.9 & - \\
NLAF-PCA [34] & 74.18 & 0.049 & 0.973 & 0.999 & 5.16 & - \\
Our method & $\mathbf{9 1 . 0 4}$ & $\mathbf{0 . 0 0 7 1 4}$ & $\mathbf{0 . 9 9 9 4}$ & $\mathbf{1}$ & $\mathbf{5 . 2 9 9}$ & $\mathbf{5 . 3 7 9}$ \\
\hline
\end{tabular}

Table 3. Quantitative analysis of fusion methods for dataset 3

\begin{tabular}{ccccccc}
\hline Methodology & PSNR (in dB) & RMSE & CC & SSIM & Entropy & MI \\
\hline PCA [26] & 54.47 & 0.48 & 0.895 & 0.971 & 2.57 & - \\
SWT [21] & 65.05 & 0.140 & 0.854 & 0.984 & 1.20 & - \\
DWT [20] & 65.08 & 0.141 & 0.8545 & 0.9848 & 1.21 & - \\
FDCT [27] & 66.59 & 0.119 & 0.8953 & 0.9858 & 3.86 & - \\
SWT+PCA [35] & 67.45 & 0.10 & 0.912 & 0.987 & 3.9 & - \\
NLAF-PCA [34] & 69.18 & 0.089 & 0.93 & 0.99 & 3.95 & - \\
Our method & $\mathbf{7 5 . 4 6}$ & $\mathbf{0 . 0 4 2 9}$ & $\mathbf{0 . 9 8 7}$ & $\mathbf{0 . 9 9 9 8}$ & $\mathbf{4 . 3 2}$ & $\mathbf{4 . 2 1}$ \\
\hline
\end{tabular}

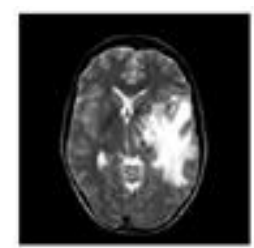

(a)

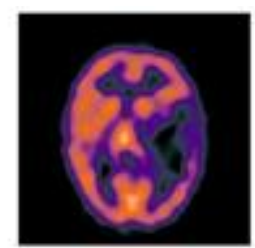

(b)

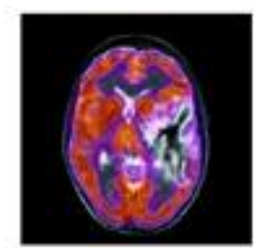

(c)
Figure. 6 RGB fused image with proposed IGNLA filtering process (a) MR-T2 (b) SPECT-Tc (c) Fused image

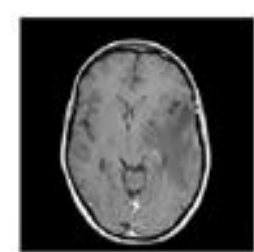

(a)

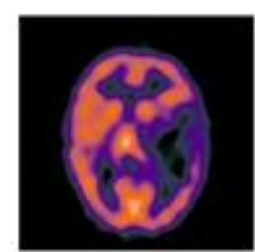

(b)

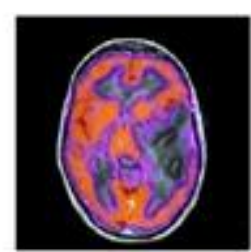

(c)
Figure. 7 RGB fused images with proposed IGNLA filtering process (a) MR-Gad (b) SPECT-Tc (c) Fused image

Table 4. Quantitative analysis of fusion methods for MRGad, MR-T2 and SPECT-Tc images with proposed fusion

\begin{tabular}{ccccc} 
Combination & Entropy & Mean & $\begin{array}{c}\text { Standard } \\
\text { Deviation }\end{array}$ & $\begin{array}{c}\text { Running } \\
\text { time } \\
(\mathrm{sec})\end{array}$ \\
\hline $\begin{array}{c}\text { MR-T2 \& } \\
\text { SPECT-Tc }\end{array}$ & 4.31 & 44.20 & 66.72 & 6.32 \\
$\begin{array}{c}\text { MR-Gad \& } \\
\text { SPECT-Tc }\end{array}$ & 4.50 & 53.299 & 72.12 & 5.85 \\
\hline
\end{tabular}

\section{Conclusion}

This article innovated a new texture preserving fusion approach for medical images using IGNLA filtering that combined both Guided filter and nonlinear anisotropic filtering for extracting the base or approximate and detail layers from the source medical images to be fused. Image statistics based fusion rule is utilized to obtain a fused image from the extracted layers of source medical images while preserving the texture information. Quality of fused images is evaluated with respect to the image quality assessment metrics and compared the same with existing fusion approaches discussed in the literature. Further, proposed medical image fusion approach is extended for color images and tested with MR-Gad, MR-T2 and SPECT-Tc images. In addition, several quality metrics are computed to demonstrate visual and quantitative evaluation of fused color images.

\section{References}

[1] P. J. Burt and E. H. Adelson, "The Laplacian Pyramid as a Compact Image Code", IEEE Transactions on Signal Processing, Vol. 31, No. 4, pp. 532-540, April 1983.

[2] K. Sharmila, S. Rajkumar, and V. Vijayarajan, "Hybrid method for Multimodality Medical image fusion using Discrete Wavelet Transform and Entropy concepts with Quantitative Analysis", In: Proc. of International Conf. on Communication and Signal Processing, 2013. 
[3] J. Nunez, X. Otazu, O. Fors, A. Prades, V. Pala and R. Arbiol, "Multiresolution Based Image Fusion with Additive Wavelet Decomposition", IEEE Transactions on Geoscience and Remote Sensing, Vol. 37, No. 3, pp.4-11, 1999.

[4] V. S. Petrović and C. S. Xydeas, "Gradientbased multiresolution image fusion", IEEE Transactions on Image Processing, Vol. 13, No. 2, pp. 228-237, Feb 2004.

[5] P. Suetens, "Fundamentals of medical imaging", Cambridge: Cambridge University Press; 2009.

[6] K. Zhan, Y. Xie, H. Wang, and Y. Min, "Fast filtering image fusion", Journal of Electronic Imaging, Vol. 26, No. 6, pp. 063004(1-18), 2017.

[7] B. Yang and S. Li, "Multi-focus image fusion based on spatial frequency and morphological operators", Chinese Optical Letters, Vol. 5, pp. 452-453, 2007.

[8] D. Fasbender, J. Radoux and P. Bogaert, "Bayesian data fusion for adaptable image pansharpening", IEEE Transactions on Geoscience and Remote Sensing, Vol. 46, pp. 1847-1857, 2008.

[9] H. Khemila and C. B. Rhaimi, "Bayesian Fusion: Application in Medical Imaging", In: Proc. of the $17^{\text {th }}$ International Conf. on Science and Techniques of Control and Computer Engineering, pp. 87-92, 2016.

[10] R. Shen, I. Cheng, J. Shi, and A. Basu, "Generalized random walks for fusion of multiexposure images", IEEE Transactions on Image Processing, Vol. 20, pp. 3634-3646, 2011.

[11] M. Xu, H. Chen, and P. K. Varshney, “An image fusion approach based on Markov random fields", IEEE Transactions on Geoscience and Remote Sensing, Vol. 49, pp. 5116-5127, 2011.

[12] E. A. Newman and P. H. Hartline, "Integration of visual and infrared information in bimodal neurons of the rattlesnake optic tectum", Science, Vol. 213, No. 4509, pp. 213-789, 1981.

[13] A. M. Waxman, M. Aguilar, D. A. Fay, D. B. Ireland, and J. P. Racamato, "Solid-state color night vision: Fusion of low-light visible and thermal infrared imagery", Lincoln Lab J., Vol. 11, pp. 41-60, 1998.

[14] N. S. Kumar and R. Prabhakaran, "A Novel Approach for High Intension Image with Gradient Pyramid", SSRG International Journal of Mobile Computing Application, Vol. 4, No. 3, pp. 13-17, 2017.

[15] J. Sun, Q. Han, L. Kou, L. Zhang, K. Zhang, and Z. Jin, "Multi-focus Image Fusion Based on Laplacian Pyramids", Journal of Optical Society of America A, Vol. 35, No. 3, pp. 480490, 2018.

[16] A. Toet, "Image Fusion by a Ratio of Low-pass Pyramid", Pattern Recognition Letters, Vol. 9, No. 4, pp. 245-253, 1989.

[17] C. Sahu and R. K. Sahur, "Pyramid based Image Fusion", International Journal of Engineering and Computer Science, Vol. 3, No. 8, pp. 7890-7894, 2014.

[18] N. Uniyal and S. K. Verma, "Image Fusion using Morphological Pyramid Consistency Method", International Journal of Computer Applications, Vol. 95, No. 25, pp. 34-38, 2014.

[19] V. Bhavana and H. K. Krishnappa, "Multimodality Medical Image Fusion Using Discrete Wavelet Transform", In: Proc. of the $4^{\text {th }}$ International Conf. on Eco-friendly Computing and Communication Systems, Vol. 70, pp. 625631, 2015.

[20] M. B. Abdulkareem, "Design and development of multimodal medical image fusion using discrete wavelet transform", In: Proc. of International Conf. on Inventive Communication and Computational Technologies, pp. 1629-1633, 2018.

[21] O. Prakash and A. Khare, "CT and MR Images Fusion Based on Stationary Wavelet Transform by Modulus Maxima", Computational Vision and Robotics, Advances in Intelligent Systems and Computing, Vol. 332, pp. 199-204, 2015.

[22] P. Chai, X. Luo and Z. Zhang, "Image Fusion Using Quaternion Wavelet Transform and Multiple Features", IEEE Access, Vol. 5, pp. $6724-6734,2017$.

[23] A. R. Sanjay, R. K. Soundrapandiyan, M. Karuppiah, and R. Ganapathy, "CT and MRI Image Fusion Based on Discrete Wavelet Transform and Type-2 Fuzzy Logic" International Journal of Intelligent Engineering and Systems, Vol. 10, No. 3, pp. 355-362, 2017.

[24] S. S. Chavan, A. Mahajan, S. N Talbar, S. Desai, M. Thakur, and A. Dcruz "Nonsubsampled rotated complex wavelet transform (NSRCxWT) for medical image fusion related to clinical aspects in neurocysticercosis", Computers in Biology and Medicine, Vol. 81, No. 1, pp. 64-78, 2017.

[25] E. Daniel, J. Anitha and J. Gnanaraj "Optimum Laplacian wavelet mask based medical image using hybrid cuckoo search - grey wolf optimization algorithm", Knowledge-Based Systems, Vol. 131, No. 1, pp. 58-69, 2017.

[26] M. Pan, J. Jiang, F. Zhang, and Q. Rong, "Medical Image Registration and Fusion Using 
Principal Component Analysis", The International Arab Journal of Information Technology, Vol. 14, No. 4, pp. 512-520, 2017.

[27] B. Sudharani, A. V. Kiranmai, and N. Suguna, "Image Fusion Using Fast Discrete Curvelet Transform", International Journal of Computer Science, Mathematics and Engineering, Vol. 3, No. 12, pp. 7-9, 2016.

[28] S. Li, X. Kang, and J. Hu, "Image fusion with guided filtering", IEEE Transactions on Image Processing: A Publication of the IEEE Signal Processing Society, Vol. 22, No. 7, pp. 28642875, 2013.

[29] Z. Farbman, R. Fattal, D. Lischinski, and R. Szeliski, "Edge-preserving decompositions for multi-scale tone and detail manipulation", In ACM Transactions on Graphics, Vol. 27, No. 3, 2008.

[30] Y. Jiang and M. Wang, "Image fusion using multiscale edge preserving decomposition based on weighted least squares filter", IET Image Processing, Vol. 8, No. 3, pp.183-190, 2014.

[31] K. P. Upla, S. Joshi, and M. C. Patel, "An edge preserving multi-resolution image fusion: Use of joint bilateral filter", In: Proc. of IEEE International Symposium on Geoscience and Remote Sensing, 2014.

[32] B. K. S. Kumar, "Image fusion based on pixel significance using cross bilateral filter", Signal, Image and Video Processing, Vol. 9, No. 5, pp. 1193-1204, 2015.

[33] F. Kahraman, C. D. Mendi, and M. Gokmen, "Image frame fusion using $3 \mathrm{~d}$ anisotropic diffusion", In: Proc. of the $23^{\text {rd }}$ International Symposium on Computer and Information Sciences, pp. 1-6, 2008.

[34] S. Rikhari and S. Jaiswal, "MR and CT Image Fusion Using Nonlinear Anisotropic Filtering in PCA", Journal of Advanced Research in Dynamic and Control Systems, Vol. 3, pp. 790797, 2018.

[35] S. Aymaz and C. Kose, "Multi-Focus Image Fusion Using Stationary Wavelet Transform (SWT) and Principal Component Analysis (PCA)", In: Proc. of the $10^{\text {th }}$ International Conf. on Electrical and Electronics Engineering, pp. 1176-1180, 2018.

[36] P. Perona and J. Malik, "Scale-space and edge detection using anisotropic diffusion", IEEE Transactions on Pattern Analysis and Machine Intelligence, Vol. 12, No. 7, pp. 629-639, 1990. 